\title{
Exploring the Risk-Reward Balance in Focal Therapy for Prostate Cancer A Contribution to the Debate
}

Anouck Kluytmans, Jurgen Fütterer, Mark Emberton, Michiel Sedelaar \& Janneke Grutters

\section{AUTHOR DETAILS}

Anouck Kluytmans

Department of Health Evidence, Radboud Institute for Health Sciences, Radboudumc

Jurgen Fütterer

Department of Radiology and Nuclear Medicine, Radboud Institute for Molecular Life Sciences, Radboudumc

Mark Emberton

Professor of Interventional Oncology, Division of Surgical and Interventional Science, University College London

Michiel Sedelaar

Department of Urology, Radboud Institute for Health Sciences, Radboudumc

Janneke Grutters

Departments of Health Evidence and Operating Rooms, Radboud Institute for Health Sciences, Radboudumc

TAKE HOME MESSAGE (40/40 words, upload as separate file)

Given the burden of toxicity that is associated with the whole-gland treatment of localised prostate cancer ( $\mathrm{PCa}$ ), some patients might be willing to consider treatments that trade-off a degree of disease progression against a higher likelihood of preserving genito-urinary function.

120-CHARACTER SUMMARY FOR EU'S TWITTER

Radical treatment is not free from harm; a contribution to the debate surrounding focal therapy for prostate cancer. 
Focal therapy (FT) for the treatment of localized prostate cancer offers an alternative treatment strategy for men seeking active treatment. Although relatively new, existing studies suggest that the majority of men who undergo FT tend to maintain levels of genito-urinary function that are indistinguishable from their pre-treatment status. However, as part of the shared decision making process, men need to balance good tolerability against a greater risk of recurrence given that much of the prostate remains intact after FT. In order to explore this trade-off, we used decision modelling. Our findings show that the burden of toxicity associated with radical prostatectomy (RP) is considerable, as an average of 243 days of perfect health are lost per patient due to treatment-induced urinary incontinence and erectile dysfunction. Given this effectiveness gap in current care, we explored by how much mortality - as worst-case outcome of disease progression - could increase to still result in net health benefit. To do this we mapped the net health benefit/loss of FT, in comparison to RP, for different levels of function preservation and increases in mortality. We believe our modelling exercise might help inform future studies that seek to enhance our understanding of how men make treatment decisions.

\section{KEYWORDS}

Prostate cancer; focal therapy; burden of toxicity; genito-urinary function; radical prostatectomy MAIN TEXT (995/1000 words)

In 2018, the European Association of Urology (EAU) published a position paper on focal therapy (FT) in primary localised prostate cancer (PCa) [1]. The association states that FT can only be considered beneficial to patients in the long run if it provides fewer functional complications and at least equivalent survival efficacy, compared to current care. However, whole-gland approaches to PCa, such as radical prostatectomy (RP), are known to result in functional complications for a significant number of patients, impairing their quality of life for the rest of their (often relatively long) lives. Consequently, if it is true that FT results in fewer functional complications (a reward), perhaps some men are willing to trade-off a degree of increased probability (versus standard care) of disease progression (a risk). Such a trade-off has an inherent temporal element in that treatment-related toxicity is both inevitable and immediate whereas any compromise in oncological outcome is going to be deferred and will be confined to a minority. In this brief correspondence we will model existing evidence on RP to explore 1) how much health is lost to functional complications associated with RP, and 2) by how much mortality - as worstcase outcome of disease progression - could increase to still result in net health benefit.

Let us assume a hypothetical target population of PCa patients whose risk profile makes them eligible for both RP and FT (e.g. men with PSA $\leq 15 \mathrm{ng} / \mathrm{mL}$ and a maximum Gleason $\leq 3+4$ that is unilateral [6]). In modelling complication-related versus disease-related losses in health, we are interested in both the quality and duration of patients' lives. The quality-adjusted life-year (QALY) provides a metric that corrects the duration of patients' lives for the quality (utility) in which that life is spent - allowing for trade-offs [7]. For example, 10 QALYs means both 10 life-years spent in perfect health (utility of 1 ) and 20 life-years spent in mediocre health (utility of 0.5 ). 
We used decision modelling to synthesize the available evidence and quantify the current loss in QALYs due to functional complications after RP [8]. The model structure is shown in Appendix Figure 1 and 2. Following treatment, patients may or may not experience functional complications. These complications are associated with reduced quality of life. The model then simulated yearly cycles in which patients remained in their starting health state, improved (at year 2), or died - all with associated quality of life. At the end of the lifetime horizon, the average QALYs were calculated.

Regarding functional complications, we focused on urinary incontinence (UI) and erectile dysfunction (ED) though we appreciate there are others. We used two studies that reported UI and ED rates after robot-assisted RP specifically $[2,3]$. Since we expected the UI and ED rates to contain overlap, we estimated the probability that patients have both UI and ED (see Appendix Table 1). We found quality of life estimates for having UI, ED, and UI and ED both [4]. Regarding survival, we used 18-year survival data provided by Bill-Axelson et al to estimate mortality after RP [9]. See Appendix Table 2 for an overview of all parameters estimates and their sources.

Assuming a starting age of 65, our model shows that patients, on average, experience 14.90 QALYs after RP. If UI and ED is prevented completely, ceteris paribus, this results in 15.56 QALYs. In other words, an average of 0.66 years (i.e. 243 days) in perfect health are foregone per patient due to functional complications after RP.

Part of this QALY loss could potentially be saved by less aggressive treatment strategies, such as FT. If FT improves functional complications, perhaps some increase in disease progression is tolerable. However, disease progression following FT remains unknown, as long-term studies are unavailable. Therefore, we simulated at which relative risk (RR) of mortality the QALYs gained by preventing all complications no longer outweigh the increased risk of mortality. Here, mortality serves as a worst-case end result of disease progression. We assumed this RR to apply for the first 18 years. The point of QALY indifference - where the benefits of preventing functional complications no longer outweigh an increase in mortality - lies at a 15\% increase in mortality compared to RP (RR 1.15). In other words, the 243 days of perfect health that are lost to functional complications after RP are 'worth' an increase in mortality of up to $15 \%$.

However, it is unlikely that FT eliminates functional complications completely. We therefore investigated the point of QALY indifference for various rates of mortality increase and function preservation. When FT prevents $50 \%$ of all functional complications seen after RP, the yearly relative risk of dying should remain below 1.075 (i.e. $7.5 \%$ increase in mortality) to result in expected health benefit (Figure 1). If FT prevents 70\% of complications, this increases to a RR of 1.1. See Appendix Figures 3 and 4 for similar results when only UI or ED were considered, respectively. 


\begin{tabular}{|c|c|c|c|c|c|c|c|c|}
\hline+ & $100 \%$ & 243 & 202 & 162 & 122 & 83 & 45 & 6 \\
\hline ญ & $90 \%$ & 217 & 177 & 137 & 97 & 58 & 20 & -19 \\
\hline ్ㅡㄹ & $80 \%$ & 192 & 152 & 112 & 73 & 34 & -5 & -43 \\
\hline 등 & $70 \%$ & 167 & 127 & 87 & 48 & 9 & -29 & -67 \\
\hline ᄃ & $60 \%$ & 142 & 103 & 63 & 24 & -15 & -53 & -91 \\
\hline 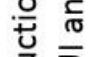 & $50 \%$ & 118 & 78 & 39 & 0 & -39 & -77 & -115 \\
\hline 공 & $40 \%$ & 94 & 54 & 15 & -24 & -62 & -100 & -138 \\
\hline 党 & $30 \%$ & 70 & 31 & -9 & -47 & -86 & -123 & -161 \\
\hline 물 & $20 \%$ & 46 & 7 & -32 & -70 & -109 & -146 & -184 \\
\hline.$\stackrel{\underline{0}}{\pi}$ & $10 \%$ & 23 & -16 & -55 & -93 & -131 & -169 & -206 \\
\hline$\stackrel{\circ}{\xi}$ & $0 \%$ & 0 & -39 & -78 & -116 & -154 & -192 & -228 \\
\hline $\begin{array}{l}0 \\
\text { o̊ }\end{array}$ & & 1.000 & 1.025 & 1.050 & 1.075 & 1.100 & 1.125 & 1.150 \\
\hline
\end{tabular}

Relative risk of dying - compared to RP

Figure 1. Exploratory analysis of health benefit/loss of a hypothetical focal treatment compared to radical prostatectomy. On the $x$-axis, the relative risk of dying, when comparing FT to RP, is varied from 1 (equal mortality rates) to 1.15 (15\% increase in mortality rates). On the $y$-axis, the relative reduction in both urinary incontinence and erectile dysfunction is varied from $0 \%$ (no complication reduction compared to RP) to $100 \%$ (FT preventing all RP complications). Green numbers indicate the number of days in perfect health gained by FT, red numbers indicate the number of days in perfect health lost by FT. The zeros in black indicate points of indifference, where FT and RP result in equal health.

Note that all of the above was calculated without considering a specific focal modality. Although preliminary, the findings synthesized by Valerio et al do provide gross estimates of the complications after, for example, focal HIFU [10]. If, as reported there, focal HIFU prevents UI by $88 \%$ and ED by $84 \%$, the point of QALY indifference lies at a $13 \%$ increased risk of dying.

The implication of this brief commentary is that current care leaves much to be desired in terms of toxicity profile. RP is associated with functional complications that affect patients' quality of life quite considerably. Therefore, some level of risk (here: increased mortality) might be tolerable when considering focal innovations that could reduce the burden of complications. In reality, both PCa itself and treatment trajectory are more complex than we were able to incorporate in our model. We intend to contribute to the debate surrounding FT by visualizing its window of opportunity based on the shortcomings of current treatment. Of course, ultimately, shared decision-making between doctor and patient may facilitate a personal value trade-off between expected duration and quality of life.

\section{ACKNOWLEDGEMENT}

Mark Emberton receives research support from the United Kingdom's National Institute of Health Research (NIHR) UCLH/UCL Biomedical Research Centre. He has been an NIHR Senior Investigator since 2014. 


\section{Appendix Figure 1 - Decision tree after treatment}

Visual presentation of our model, part 1. The target population consisted of patients with primary localised PCa. Following either radical prostatectomy or a hypothetical focal treatment, patients may have 1) no functional complications, 2) urinary incontinence only, 3) erectile dysfunction only, or 4) both urinary incontinence and erectile dysfunction.

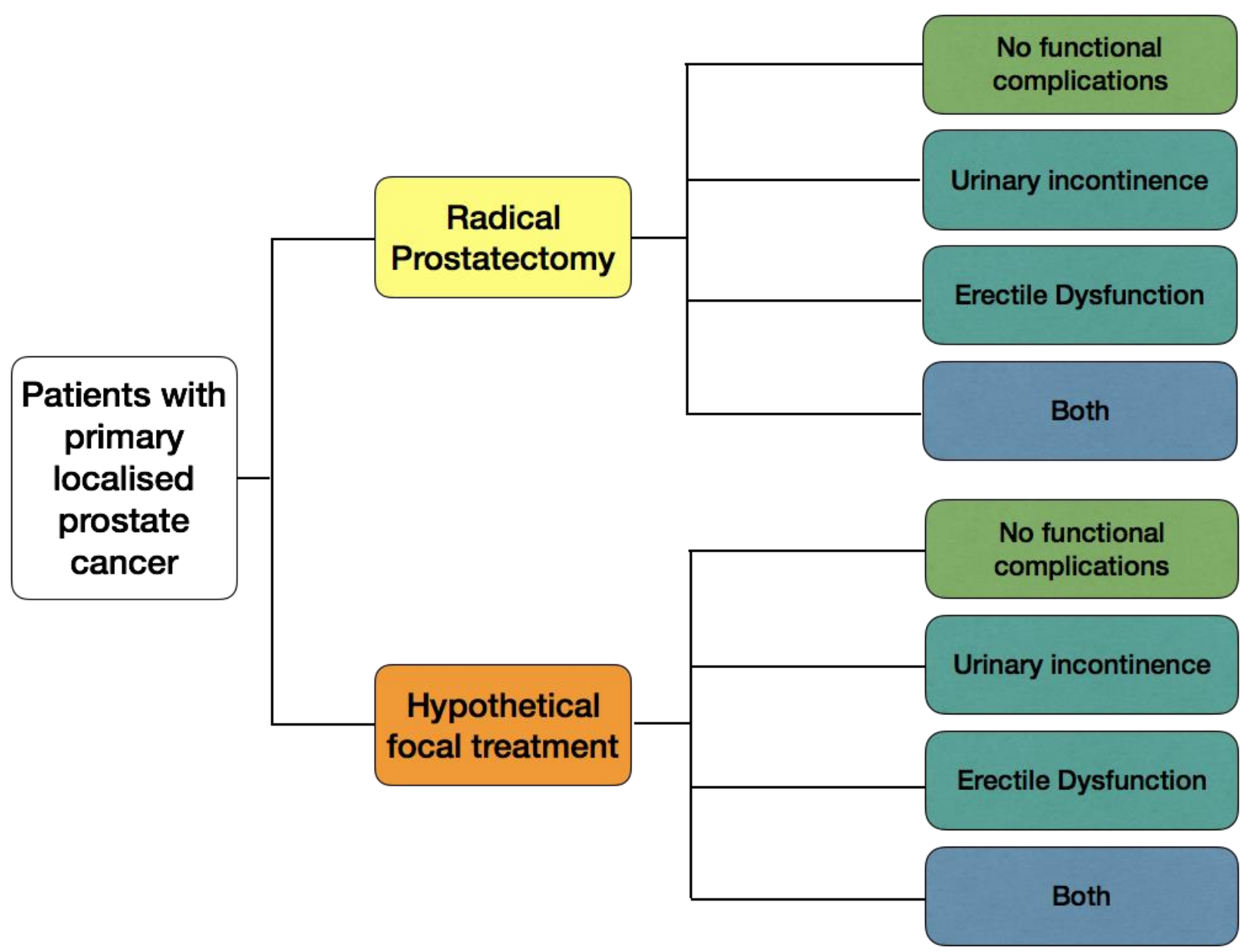




\section{Appendix Figure 2 - Long-term part of the model}

Visual presentation of our model, part 2. To extrapolate the results, the decision tree was followed by a long-term, or Markov, part in order to assess the long-term consequences. Patients either remain in their initial health state, experience function improvement (at year 2 only, dotted arrows), or die.

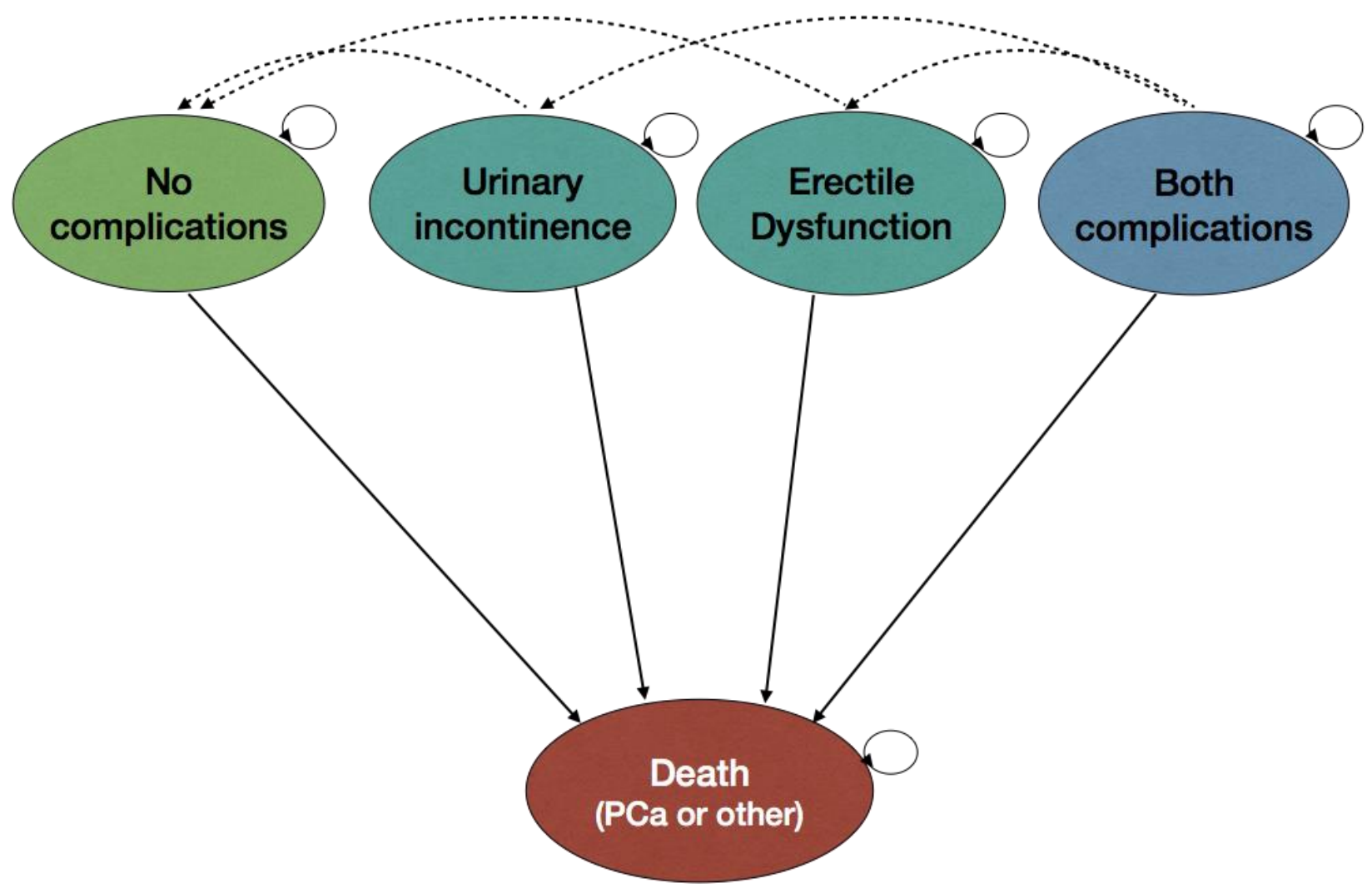




\section{Appendix Table 1 - Complication rates in the model}

This table shows how complication rates reported in literature and Appendix Table 1 were used in the model. Due to suspected overlap in rates reported for urinary incontinence and erectile dysfunction, yet lack of data on the magnitude of this overlap, we used a simple computation to estimate the probability of only urinary incontinence, only erectile dysfunction, and both urinary incontinence and erectile dysfunction. We acknowledge that the method we used is potentially flawed, as these complications are likely related. However, given lack of data on either the union or intersection of the probabilities reported in literature, the recalculation we performed seems like a viable alternative.

\begin{tabular}{|l|l|l|l|l|}
\hline & Parameter & Source & Estimate & Recalculation of probability \\
\hline \multirow{3}{*}{$\begin{array}{l}\text { Year of } \\
\text { treatment }\end{array}$} & Urinary incontinence only & Haglind 2015 & 0.213 & $0.213-\left(0.213^{*} 0.704\right)=0.063$ \\
\cline { 2 - 5 } & Erectile dysfunction only & Haglind 2015 & 0.704 & $0.704-\left(0.213^{*} 0.704\right)=0.554$ \\
\cline { 2 - 5 } & Both & - & $?$ & $0.213^{*} 0.704=0.150$ \\
\hline \multirow{3}{*}{ At year 2 } & Urinary incontinence only & Ficarra 2013 & 0.090 & $0.090-(0.090 * 0.180)=0.074$ \\
\cline { 2 - 5 } & Erectile dysfunction only & Ficarra 2013 & 0.180 & $0.180-(0.090 * 0.180)=0.164$ \\
\cline { 2 - 5 } & Both & - & $?$ & $0.090 * 0.180=0.016$ \\
\hline
\end{tabular}




\section{Appendix Table 2-Model input}

This table contains all input parameters for the model and their sources.

\begin{tabular}{l|l|l|l} 
Parameter & Estimate & Notes & Source \\
\hline $\begin{array}{l}\text { Transition probabilities } \\
\text { Probability of urinary incontinence after RP, year one }\end{array}$ & 0.213 & Based on robot-assisted RP & Haglind 2015 \\
\hline $\begin{array}{l}\text { Probability of urinary incontinence after RP, years } \\
\text { after }\end{array}$ & 0.090 & Based on robot-assisted RP & Ficarra 2013 \\
\hline Probability of erectile dysfunction after RP, year one & 0.704 & Based on robot-assisted RP & Haglind 2015 \\
\hline Probability of erectile dysfunction after RP, years after & 0.180 & Based on robot-assisted RP & Ficarra 2013 \\
\hline Probability of dying, 1-18 years after RP & - & Cycle-dependent, based on intermediate risk group & Bill-Axelson 2014 \\
\hline Probability of dying, 19-35 years after RP & - & General mortality rates for Dutch male population & Statistics \\
\hline \begin{tabular}{l|l|l|l} 
Health effects - utilities used to compute QALYs \\
Utility for having no complication
\end{tabular} & 1 & & Netherlands \\
\hline Utility for having only urinary incontinence & 0.83 & & Stewart 2005 \\
\hline Utility for having only erectile dysfunction & 0.89 & & Stewart 2005 \\
\hline Utility for having both complications & 0.79 & & Stewart 2005 \\
\hline Utility for being dead & 0 & Reference value for death health state & Stewart 2005 \\
\hline Supporting parameters in the model & 65 years & Based on average profile of patient opting for RP & - \\
\hline Starting age of the cohort & $1.5 \%$ & In compliance with Dutch guidelines & Dutch manual \\
\hline Discount rate for health effects in the model &
\end{tabular}




\section{Appendix Figure 3 - Map of outcomes for reductions in urinary incontinence only}

Similar to Figure 1 in the main text, this figure shows the average number of days in perfect health gained or lost after focal therapy, compared to radical prostatectomy. This figure focuses on reduction in urinary incontinence only, assuming that erectile dysfunction remains equal to that of RP.

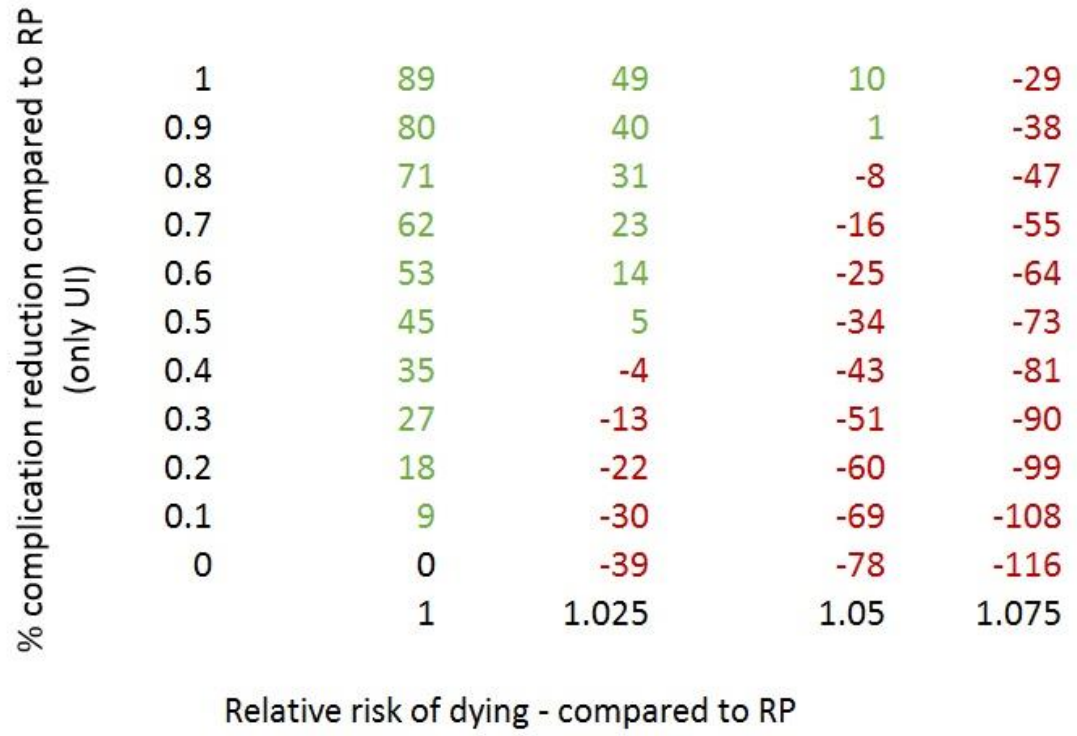

\section{Appendix Figure 4-Map of outcomes for reductions in erectile dysfunction only}

Similar to Figure 1 in the main text, this figure shows the average number of days in perfect health gained or lost after focal therapy, compared to radical prostatectomy. This figure focuses on reduction in erectile dysfunction only, assuming that urinary incontinence remains equal to that of $\mathrm{RP}$.

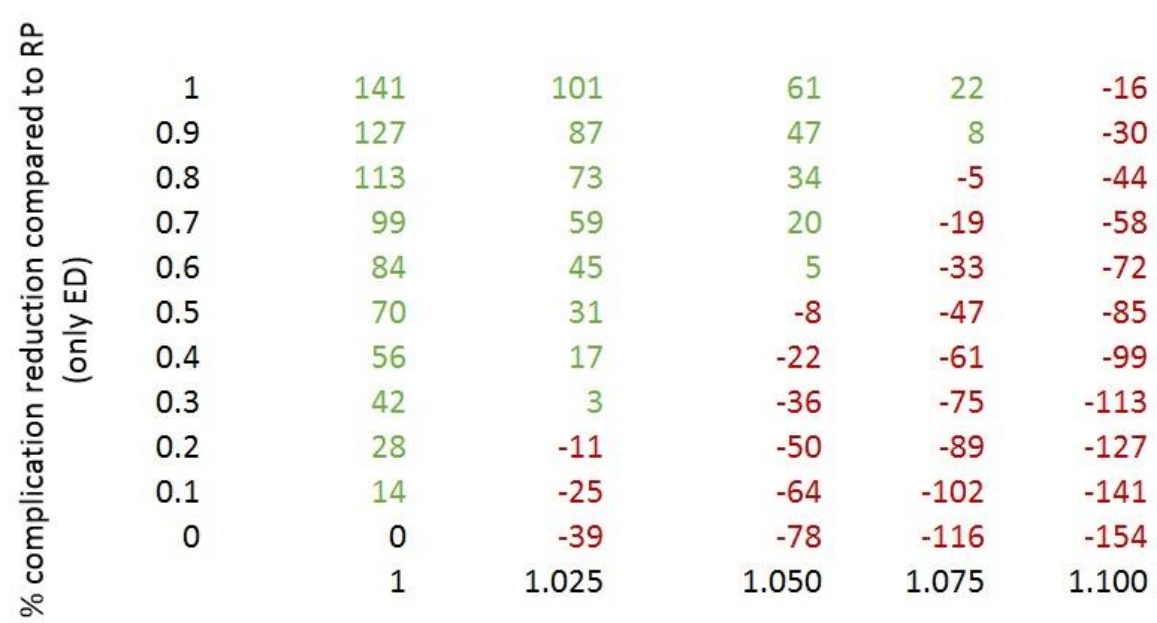




\section{References}

1. van der Poel, H.G., et al., Focal Therapy in Primary Localised Prostate Cancer: The European Association of Urology Position in 2018. Eur Urol, 2018.

2. Ficarra, V., et al., Long-term evaluation of survival, continence and potency (SCP) outcomes after robot-assisted radical prostatectomy (RARP). BJU Int, 2013. 112(3): p. 338-45.

3. Haglind, E., et al., Urinary Incontinence and Erectile Dysfunction After Robotic Versus Open Radical Prostatectomy: A Prospective, Controlled, Nonrandomised Trial. Eur Urol, 2015. 68(2): p. 216-25.

4. Stewart, S.T., et al., Utilities for prostate cancer health states in men aged 60 and older. Med Care, 2005. 43(4): p. 347-55.

5. $\quad$ Pesapane, F., et al., The prostate cancer focal therapy. Gland Surgery, 2017. 7(2): p. 89-102.

6. van den Bos, W., et al., Focal therapy in prostate cancer: international multidisciplinary consensus on trial design. European urology, 2014. 65(6): p. 1078-1083.

7. Neumann, P.J. and J.T. Cohen, Qalys in 2018-advantages and concerns. JAMA, 2018.

8. Caro, J.J., et al., Modeling good research practices-overview: a report of the ISPOR-SMDM Modeling Good Research Practices Task Force-1. Value in health, 2012. 15(6): p. 796-803.

9. Bill-Axelson, A., et al., Radical Prostatectomy or Watchful Waiting in Early Prostate Cancer. New England Journal of Medicine, 2014. 370(10): p. 932942.

10. Valerio, M., et al., New and Established Technology in Focal Ablation of the Prostate: A Systematic Review. European Urology, 2017. 71(1): p. 17-34. 